\title{
Article \\ Multiple Single Nucleotide Polymorphism Testing Improves the Prediction of Diabetic Retinopathy Risk with Type 2 Diabetes Mellitus
}

\author{
Yu-Ting Hsiao ${ }^{1}$, Feng-Chih Shen ${ }^{2,3}$, Shao-Wen Weng ${ }^{2,3} \mathbb{D}$, Pei-Wen Wang ${ }^{2,3} \mathbb{D}$, Yung-Jen Chen ${ }^{1}$ and \\ Jong-Jer Lee ${ }^{1, *(\mathbb{D})}$
}

1 Department of Ophthalmology, Kaohsiung Chang Gung Memorial Hospital and Chang Gung University College of Medicine, Kaohsiung 83301, Taiwan; yuting1008@cgmh.org.tw (Y.-T.H.); f75622@cgmh.org.tw (Y.-J.C.)

2 Division of Metabolism and Endocrinology, Department of Internal Medicine, Kaohsiung Chang Gung Memorial Hospital and Chang Gung University College of Medicine, Kaohsiung 83301, Taiwan; carcinom@cgmh.org.tw (F.-C.S.); wengsw99@adm.cgmh.org.tw (S.-W.W.); wangpw@adm.cgmh.org.tw (P.-W.W.)

3 Center for Mitochondrial Research and Medicine, Kaohsiung Chang Gung Memorial Hospital, Kaohsiung 83301, Taiwan

* Correspondence: tojjlee@cgmh.org.tw; Tel.: +886-7-7317123 (ext. 2801)

check for updates

Citation: Hsiao, Y.-T.; Shen, F.-C.; Weng, S.-W.; Wang, P.-W.; Chen, Y.-J.; Lee, J.-J. Multiple Single Nucleotide Polymorphism Testing Improves the Prediction of Diabetic Retinopathy Risk with Type 2 Diabetes Mellitus. J. Pers. Med. 2021, 11, 689. https:// doi.org/10.3390/jpm11080689

Academic Editor: José Carmelo Adsuar Sala

Received: 18 June 2021

Accepted: 19 July 2021

Published: 21 July 2021

Publisher's Note: MDPI stays neutral with regard to jurisdictional claims in published maps and institutional affiliations.

Copyright: (c) 2021 by the authors. Licensee MDPI, Basel, Switzerland. This article is an open access article distributed under the terms and conditions of the Creative Commons Attribution (CC BY) license (https:// creativecommons.org/licenses/by/ $4.0 /)$.

\begin{abstract}
Diabetic retinopathy (DR) is one of the most frequent causes of irreversible blindness, thus prevention and early detection of DR is crucial. The purpose of this study is to identify genetic determinants of DR in individuals with type 2 diabetic mellitus (T2DM). A total of 551 T2DM patients (254 with DR, 297 without DR) were included in this cross-sectional research. Thirteen T2DM-related single nucleotide polymorphisms (SNPs) were utilized for constructing genetic risk prediction model. With logistic regression analysis, genetic variations of the FTO (rs8050136) and PSMD6 (rs831571) polymorphisms were independently associated with a higher risk of DR. The area under the curve (AUC) calculated on known nongenetic risk variables was 0.704 . Based on the five SNPs with the highest odds ratio (OR), the combined nongenetic and genetic prediction model improved the AUC to 0.722. The discriminative accuracy of our 5-SNP combined risk prediction model increased in patients who had more severe microalbuminuria (AUC $=0.731$ ) or poor glycemic control (AUC = 0.746). In conclusion, we found a novel association for increased risk of DR at two T2DM-associated genetic loci, FTO (rs8050136) and PSMD6 (rs831571). Our predictive risk model presents new insights in DR development, which may assist in enabling timely intervention in reducing blindness in diabetic patients.
\end{abstract}

Keywords: diabetic retinopathy; single nucleotide polymorphism; diabetes mellitus; genetics

\section{Introduction}

Affecting more than one-third of patients with diabetes mellitus (DM), diabetic retinopathy (DR) is a common vision-threatening diabetic microvascular complication, which can lead to blindness [1,2]. DR is a major reason for vision loss in working-age individuals across the world, ranging from $3-7 \%$ in developing regions to $15-17 \%$ in developed countries [3,4]. Visual impairment has an impact that extends beyond the individual, as communities and economies lose earning productivity and a need for social support arises [5]. DR can advance to irreversible stages with very few symptoms. Moreover, patients with earlier stages of DR, such as mild or moderate non-proliferative diabetic retinopathy (NPDR), usually do not require treatment nor does it typically affect their vision, making them unaware of the condition [6]. Therefore, there is an essential need for screening and early detection to implement timely and effective interventions for DR. 
As current approaches for treating DR are most effective when the condition is caught early, ophthalmologists need an accurate way to quickly identify patients who need treatment. Annual fundus check-ups are necessary, and depending on the degree of retinopathy, the interval between fundus examinations may need to be reduced. It is challenging to keep up with the demand to provide requisite annual screenings for the diabetic population, especially in areas with a shortage of eye-care providers [7]. Although studies highlight the potential for artificial intelligence (AI)-assisted screening algorithms in DR, there are some downsides to machine learning, including differences in camera equipment and training of screening personnel to obtain images [8,9]. All in all, screening for DR is mandatory, though it may cause a heavy burden on clinical practice for ophthalmologists if there are no adequate decision tools [7].

Predictive models have been adopted based on the risk factors identified to estimate the incidence and development of DR $[10,11]$. Conventional risk factors include younger age at diagnosis, longer duration of diabetes, higher systolic blood pressure (SBP) and glycosylated hemoglobin $(\mathrm{HbA} 1 \mathrm{c})$ levels, presence of proteinuria, dyslipidemia, and increased body-to-mass index (BMI) [12,13]. However, according to observations from the Diabetes Control and Complications Trial, $\mathrm{HbA} 1 \mathrm{C}$ and the duration of diabetes account for just 11 percent of risk for developing DR, and other factors may account for the unexplained 89 percent risk variation [14]. These data imply that other components such as environmental and genetic determinants may contribute to the overall risk of the development and progression of DR [15-17].

Previous DR risk models either incorporate a multitude of laboratory variables or construct large-scale multilocus genetic risk scores [18-20]. However, a comprehensive model to predict DR with the combined effect of traditional risk factors and genetic variables in diabetic patients is limited [21]. Therefore, the objective of this study is to explore the key predictors and genetic variables to establish a multifactorial, practical risk prediction model for DR.

\section{Materials and Methods}

\subsection{Study Participants}

All T2DM patients aged 30 years or older who visited the endocrinology and metabolism outpatient clinics on a regular basis between February 2017 and June 2018 in Kaohsiung Chang Gung Memorial Hospital (KCGMH), Taiwan, were included in the research. Diagnosis of diabetes was based on medical records and $\mathrm{HbA} 1 \mathrm{c}$ readings of $6.5 \%$ or higher on repeated testing, according to the American Diabetes Association Criteria [22]. A total of 606 patients were recruited, and 55 patients who were unable to complete SNP genotyping or yearly retinal photographs were excluded. All procedures adhered to the Declaration of Helsinki and the ARVO statement on human subjects. The Institutional Review Board/Ethics Committee from the Committee of Medical Ethics and Human Experiments of CGMH, Taiwan, approved of the study protocol (IRB approval no. 201601206B0C501), and all individuals gave their informed consent.

A standardized interview with each patient was conducted to collect the demographic information and medical history. The hypertension guideline established by the American College of Cardiology and the American Heart Association (ACC/AHA) in 2016 was used for blood pressure measurement [23]. Measurements were taken after the patient sat silently for $5 \mathrm{~min}$ with a digital automatic blood pressure device. The participant's blood pressure was determined using the average of two measurements taken on two different occasions. The waist circumference was assessed from above the right iliac crest at the mid-axillary line, following the method in National Health and Nutrition Examination Survey (NHANES) [24]. Blood samples were collected by venipuncture for serological tests, including $\mathrm{HbA1c}$, low-density lipoprotein (LDL), and high-density lipoprotein (HDL), triglycerides, and total cholesterol. Albuminuria was sorted into 3 categories based on the urine albumin-to-creatinine ratio (UACR) collected from the first voiding of urine in the morning. According to the Kidney Disease: Improving Global Outcomes (KDIGO), 
normoalbuminuria (A1) was defined as UACR less than $30 \mathrm{mg} / \mathrm{g}$, microalbuminuria (A2) as UACR of between 30 and $<300 \mathrm{mg} / \mathrm{g}$, and macroalbuminuria (A3) as UACR greater than $300 \mathrm{mg} / \mathrm{g}$ [25].

DR was assessed by CR-2 digital non-mydriatic retinal camera (Canon, Tokyo, Japan). Both eyes were recorded with two retinal photographs centered on the macula. The presence of DR was determined by the agreement of fundus photo reading between two masked, trained ophthalmologists (YTH and JJL), and was assessed by a third ophthalmologist (YJC) if there was any insufficiency in the grading of the same photograph. Grading was based on the characteristic lesion present in either eye (i.e., cotton wool spots, microaneurysms, hard exudates, hemorrhages, venous beading, neovascularization, and intraretinal microvascular abnormalities), as defined by the International Clinical Diabetic Retinopathy (ICDR) classification [26]. DR severity was based on the worse eye and graded as follows: no apparent retinopathy, mild NPDR (microaneurysms only), moderate NPDR (more than only microaneurysms but less than severe NPDR), severe NPDR (more than twenty intraretinal hemorrhages in each of four quadrants, venous beading in more than two quadrants, intraretinal microvascular abnormalities in at least one quadrant), PDR (presence of neovascularization, vitreous/preretinal hemorrhage). Vision-threatening DR (VTDR) was characterized on the basis of severe NPDR, PDR, or clinically significant macular edema (CSME), as per the Eye Diseases Prevalence Research Group definition [27].

\subsection{SNP Genotyping}

The genetic material was isolated from leukocytes in peripheral blood samples. DNA was extracted with the Gentra Puregene kit (Qiagen, Venlo, The Netherlands). We selected a list of single nucleotide polymorphisms (SNPs) that have previously been linked to type 2 diabetes in an Asian/Han population. Genotyping of selected SNPs of the following genes: CDKAL1 (rs10946398), CDKN2A (rs10811661), FTO (rs8050136), HHEX (rs1111875), IGF2BP2 (rs4402960), IRS1 (rs2943641), KCNJ11 (rs5219), SLC22A1 (rs622342), TCF7L2 (rs7901695), KCNQ1 (rs2237892), VPS13C/C2CD4A/C2CD4B (rs7172432), SLC30A8 (rs13266634), PSMD6 (rs831571) was performed with TaqMan assay, obtained from Topgen Biotechnology (Kaohsiung City, Taiwan). The following cycling conditions were used for PCR amplification: initial denaturation $\left(95^{\circ} \mathrm{C}\right.$ for $5 \mathrm{~min}$, then $30 \mathrm{~s}$ at $\left.60^{\circ} \mathrm{C}\right), 40$ cycles of denaturation $\left(95^{\circ} \mathrm{C}\right.$ for $\left.3 \mathrm{~s}\right)$, annealing $\left(60^{\circ} \mathrm{C}\right.$ for $\left.40 \mathrm{~s}\right)$, extension $\left(60^{\circ} \mathrm{C} 30 \mathrm{~s}\right)$, and final extension $\left(72{ }^{\circ} \mathrm{C}\right.$ for $10 \mathrm{~min}$ ). On the StepOnePlus Real Time PCR System (Thermo Fisher Scientific, Waltham, MA, USA), real-time PCR was performed using 2X AceQ Probe High ROX qPCR Master Mix (Topgen Biotechnology). Genotype-calling for allelic discrimination was executed by StepOne software v2.3 (Applied Biosystems, Grand Island, NY, USA).

\subsection{Construction of the Combined Nongenetic and Genetic Risk Model}

We constructed our risk prediction model based on previously described multiple genetic risk models $[28,29]$. Our nongenetic risk prediction model included the following risk factors: HbA1c, DM duration, waist circumference, systolic blood pressure, albuminuria categories, total cholesterol, and triglyceride levels. Then, to consider the greater correlation of some SNPs with DR, we added SNPs one after another to the nongenetic risk prediction model to deduce which SNPs should be included. We started by adding SNPs with the highest odds ratio in our data, to the lowest, and determined whether inserting SNPs to the prediction model could improve the area under curve (AUC) when each SNP was added. Furthermore, we also evaluated to which diabetic subgroup our risk prediction model could enhance the validity of retinopathy risk assessment by ROC curves.

\subsection{Statistical Analysis}

Quantitative variables were shown as mean \pm standard deviation in descriptive analyses, while categorical data were represented as numbers and percentages. The Kolmogorov-Smirnov test was used to determine if the parameters had a Gaussian distribution. Mann-Whitney U test was employed to compare continuous variables, while 
Fisher's exact or Pearson's chi-squared $(\chi 2)$ tests were used to analyze categorical data as appropriate. The association between the 13 SNPs and the risk of DR was explored using additive, dominant, and recessive models. The difference in genotype and allele frequencies, and the interaction between 13 SNPs with DR risk were analyzed using multivariate logistic regression adjusted for waist circumference, SBP, DM duration, $\mathrm{HbA1c}$, albuminuria categories, total cholesterol, and triglycerides. Odds ratios (ORs) with 95\% confidence intervals (CIs) were obtained for independently associated genetic variants. To better investigate the significant differences between DR and genetic variants in DR and non-DR groups, multivariable logistic regression analyses further adjusting for DM medication usage was performed. We conducted a post hoc power analysis to calculate the statistical power using the $G^{*}$ Power 3.1.9 (from Heinrich-Heine-Universität Düsseldorf, Franz Faul, Universitat Kiel, Kiel, Germany), assuming a 2-tailed test at 5\% alpha level.

To evaluate the discriminating ability of our risk prediction model for DR patients, we calculated the area under the receiver-operating characteristic (ROC) curve. According to the method of Hanley and McNeil [30], we compared the AUCs of different nongenetic and genetic risk prediction models. All statistical analyses were performed using SPSS 20.0 (SPSS Inc., Chicago, IL, USA). A $p$ value of $<0.05$ was considered as significant.

\section{Results}

\subsection{Subject Characteristics}

In our study, a total of 551 participants with T2DM were included in the analysis, with $254(46 \%)$ patients being diagnosed with DR. Fifty-five subjects were excluded due to insufficient SNP genotyping data or fundus photographs. No significant differences were found in the characteristics of either the included or excluded group of participants except for waist circumference $(90.92 \pm 0.46 \mathrm{~cm}$ vs. $94.72 \pm 1.58 \mathrm{~cm}, p=0.01)$. The comparisons of baseline demographic data between the DR and non-DR group are shown in Table 1. Participants with DR were older, female, and had larger waist circumferences overall. The DR group had significantly higher levels of SBP $(p=0.002)$ and HbA1c $(p<0.001)$, longer duration of DM $(p<0.001)$, and worse albuminuria $(p=0.02)$. The difference of lipid profiles between the two groups was insignificant.

Table 1. Baseline characteristics of patients.

\begin{tabular}{|c|c|c|c|}
\hline & Non-DR & DR & $p$-Value \\
\hline$n$ & 297 & 254 & \\
\hline Age (year) & $60.78 \pm 9.81$ & $61.26 \pm 9.71$ & $0.648^{\mathrm{a}}$ \\
\hline Male sex, no. (\%) & $155(53.4)$ & $142(54.4)$ & $0.822^{b}$ \\
\hline Waist circumference $(\mathrm{cm})$ & $90.30 \pm 10.38$ & $91.64 \pm 11.29$ & $0.143^{\mathrm{a}}$ \\
\hline $\mathrm{SBP}(\mathrm{mmHg})$ & $136.56 \pm 18.80$ & $140.90 \pm 19.86$ & $0.002^{\mathrm{a}}$ \\
\hline $\mathrm{DBP}(\mathrm{mmHg})$ & $77.53 \pm 11.89$ & $78.41 \pm 12.49$ & $0.375^{a}$ \\
\hline Duration of DM (year) & $14.70 \pm 6.47$ & $19.15 \pm 8.73$ & $<0.001^{\mathrm{a}}$ \\
\hline $\operatorname{HbA} 1 \mathrm{c}(\%)$ & $7.24 \pm 0.88$ & $7.72 \pm 1.14$ & $<0.001^{\mathrm{a}}$ \\
\hline Total cholesterol (mg/dL) & $168.98 \pm 1.75$ & $167.27 \pm 2.07$ & $0.305^{a}$ \\
\hline $\mathrm{HDL}(\mathrm{mg} / \mathrm{dL})$ & $47.42 \pm 0.70$ & $46.89 \pm 0.89$ & $0.090^{\mathrm{a}}$ \\
\hline LDL (mg/dL) & $89.94 \pm 1.32$ & $90.18 \pm 1.58$ & $0.601^{\mathrm{a}}$ \\
\hline Triglycerides (mg/dL) & $139.92 \pm 5.26$ & $134.51 \pm 4.81$ & $0.678^{\mathrm{a}}$ \\
\hline Albuminuria categories ${ }^{\mathrm{c}}$ & & & $0.02^{b}$ \\
\hline A1, no. $(\%)$ & $176(59.3)$ & $121(47.6)$ & \\
\hline A2, no. $(\%)$ & $102(34.3)$ & $101(39.8)$ & \\
\hline A3, no. (\%) & $19(6.4)$ & $32(12.6)$ & \\
\hline
\end{tabular}

${ }^{a}$ Mann Whitney U test; ${ }^{b}$ Chi-square test; ${ }^{c}$ data from Kidney Disease: Improving Global Outcomes (KDIGO) 2012 Clinical Practice Guideline. DR: diabetic retinopathy; SBP: systolic blood pressure; DBP: diastolic blood pressure; DM: diabetes mellitus; HDL: high-density lipoprotein; LDL: low-density lipoprotein. 


\subsection{Association between SNPs and DR Risk}

The association of the polymorphisms with DR are shown in Figure 1. The DR prevalence increased significantly in cases with the risk allele (A) of FTO (rs8050136). The percentage of DR was $46.5 \%, 41.3 \%$, and $83.3 \%$ in patients with CC, CA, and AA genotype, respectively $(p=0.019)$. Furthermore, patients with the TC genotype of PSMD6 (rs831571) had a lower DR prevalence (40.3\%) than those with the TT (50.6\%) and CC genotypes $(51.4 \%)$. The DR prevalence of patients with genetic variants of PSMD6 (rs831571) was significantly different $(p=0.038)$.

(a)

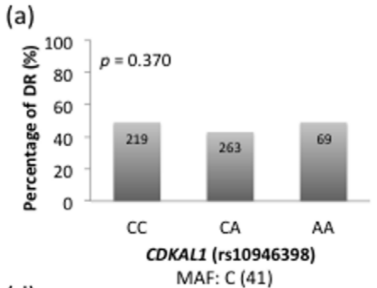

(d)

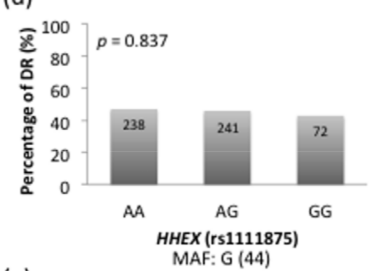

(g)

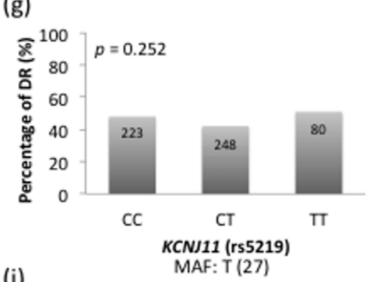

(j)
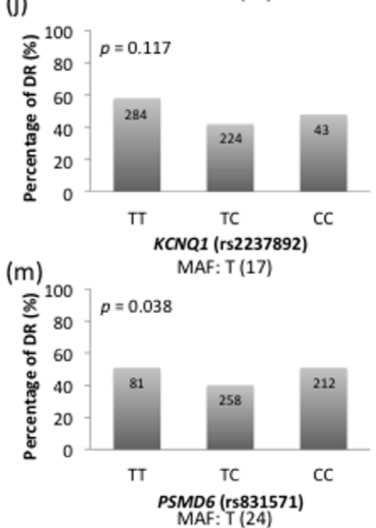

(b)

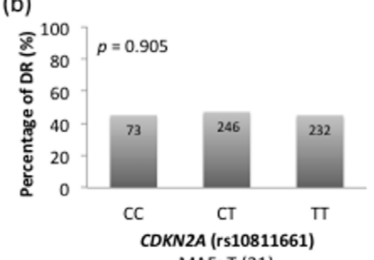

(e)

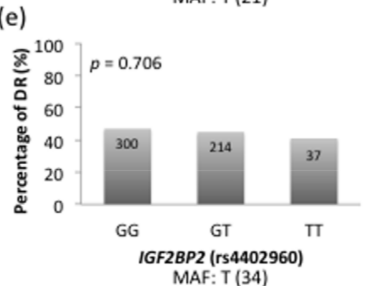

(h)

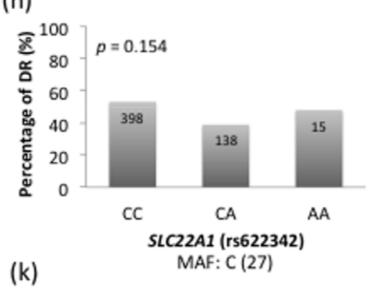

(k)
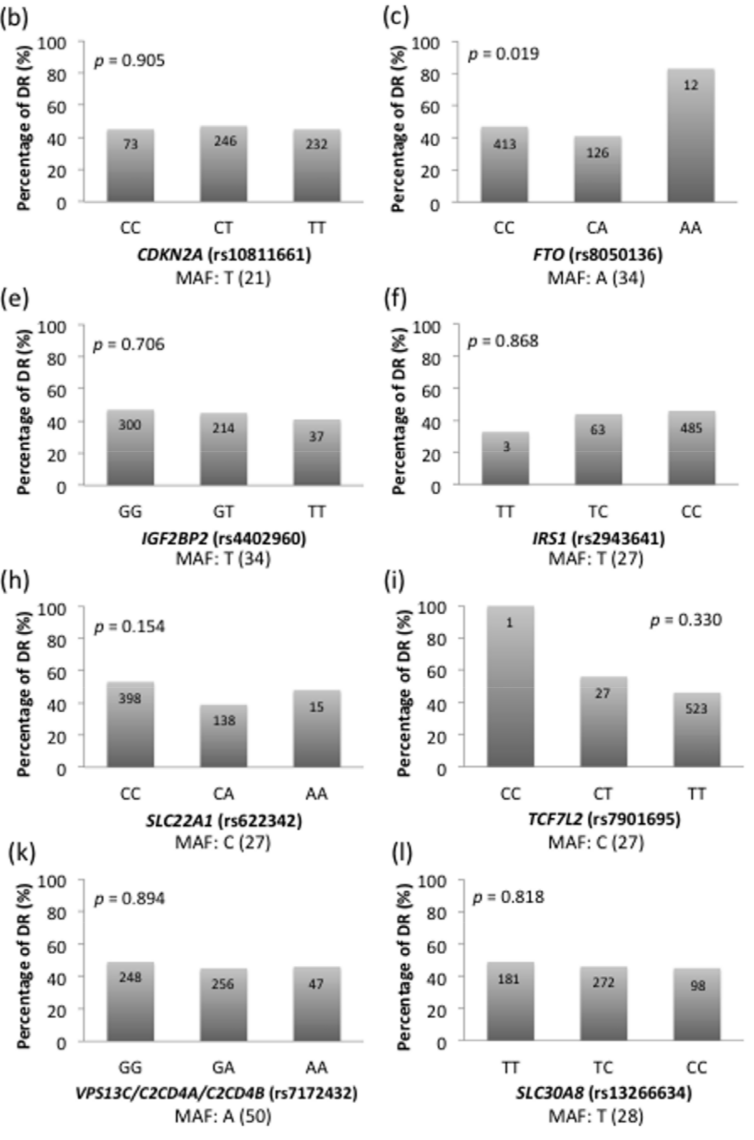

(i)
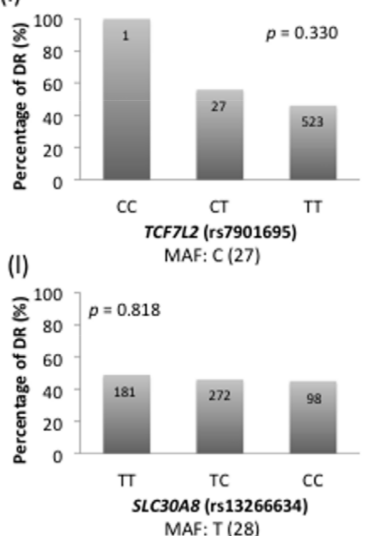

Figure 1. Comparison of diabetic retinopathy (DR) prevalence in type 2 diabetes mellitus (T2DM) patients according to 13 single nucleotide polymorphisms (SNPs) previously found to be associated with type 2 diabetes in Asian populations. (a-m) The number in the bar represents the total number of subjects in the genotype. The global minor allele frequency (MAF) of each SNP is presented at the bottom of each subfigure with percentage in brackets.

Backward stepwise logistic regression analysis of the 13 SNPs using three genetic models (additive, dominant, and recessive models) showed that FTO (rs8050136) and PSMD6 (rs831571) were independently associated with DR in the additive model (FDR-corrected $p=0.092$ and FDR-corrected $p=0.044$, respectively; Table 2) and recessive model (FDRcorrected $p=0.071$ and FDR-corrected $p=0.044$, respectively; Table 3$)$, further suggesting that the A allele in FTO (rs8050136) and C allele in PSMD6 (rs831571) were risk alleles for DR. Results from logistic regression models demonstrated that SBP (OR, 1.012; 
95\% CI, 1.002-1.022; $p=0.03)$, DM duration (OR, 1.067; 95\% CI, 1.040-1.095; $p<0.001)$, $\mathrm{HbA1c}(\mathrm{OR}, 1.288 ; 95 \%$ CI, 1.217-1.812; $p<0.001$ ), and albuminuria (OR, 1.536; 95\% CI, 0.971-1.709; $p=0.079$ ) were independently associated with DR; whereas waist circumference, total cholesterol, and triglycerides were not. After adjustment for variables including waist circumference, SBP, DM duration, $\mathrm{HbA1c}$, albuminuria categories, lipid profiles, and for DM medications, patients with FTO (rs8050136) polymorphism were significantly associated with increased DR risk (OR, 5.851; CI, 1.201-28.510; FDR-corrected $p=0.039)$, whereas those with PSMD6 (rs831571) polymorphisms were not $(p=0.076)$ (Table 4). The post-hoc power analysis was performed for the association of each SNP grouping in Tables $2-4$. The results demonstrated that the power analyses were between 0.176 and 0.359 for FTO (rs8050136), and between 0.09 and 0.659 for PSMD6 (rs831571) in the additive model (Table 2). The power analyses were 0.657 and 0.716 for $F T O$ (rs8050136) variant, and 0.647 and 0.472 for the PSMD6 (rs831571) variant in recessive models (Table 3) and when adjusted for DM medication usage (Table 4), respectively.

Table 2. Backward stepwise regression analysis on the association between SNPs and diabetic retinopathy using the additive model.

\begin{tabular}{|c|c|c|c|c|c|c|c|c|c|c|c|c|}
\hline \multirow{2}{*}{$\begin{array}{c}\text { Genetic } \\
\text { Variables }\end{array}$} & \multicolumn{3}{|c|}{ Genotypic Value } & \multicolumn{3}{|c|}{1 vs. 0} & \multicolumn{3}{|c|}{2 vs. 1} & \multicolumn{3}{|c|}{2 vs. 0} \\
\hline & 0 & 1 & 2 & S.E. & OR $(95 \% \mathrm{CI})$ & $p$-Value & S.E. & OR $(95 \%$ CI) & $p$-Value & S.E. & OR $(95 \%$ CI) & $p$-Value \\
\hline $\begin{array}{c}\text { FTO } \\
\text { (rs8050136) }\end{array}$ & $\mathrm{CC}$ & CA & AA & 1.120 & $\begin{array}{c}0.789 \\
(0.509,1.223)\end{array}$ & 0.290 & 0.829 & $\begin{array}{c}5.650 \\
(1.111,28.571)\end{array}$ & 0.044 & 3.376 & $\begin{array}{c}4.451 \\
(0.905,21.888)\end{array}$ & 0.095 \\
\hline $\begin{array}{l}\text { PSMD6 } \\
\text { (rs831571) }\end{array}$ & TT & $\mathrm{TC}$ & $\mathrm{CC}$ & 2.125 & $\begin{array}{c}0.666 \\
(0.386,1.150)\end{array}$ & 0.174 & 0.204 & $\begin{array}{c}1.692 \\
(1.135,2.525)\end{array}$ & 0.020 & 0.177 & $\begin{array}{c}1.127 \\
(0.645,1.972)\end{array}$ & 0.674 \\
\hline
\end{tabular}

Genetic variables were adjusted for waist circumference, SBP, duration of DM, albuminuria categories, HbA1c, total cholesterol, triglycerides as covariates. $p$ values were false discovery rate (FDR) corrected. Post-hoc power analysis of $F T O$ (rs8050136): 1 vs. 0 , power $=0.176 ; 2$ vs. 1 , power $=0.332 ; 2$ vs. 0 , power $=0.359$. Post-hoc power analysis of $P S M D 6(\mathrm{rs} 831571): 1$ vs. 0 , power $=0.511 ; 2$ vs. 1 , power $=0.659 ; 2$ vs. 0 , power $=0.09$. S.E.: standard error; OR: odds ratio; CI: confidence interval; vs.: versus.

Table 3. Backward stepwise regression analysis on the association between SNPs and diabetic retinopathy using the recessive model.

\begin{tabular}{ccccc}
\hline Genetic Variables & Alleles & S.E. & OR (95\% CI) & $p$-Value \\
\hline FTO (rs8050136) & CC + CA vs. AA & 3.569 & $4.605(0.944,22.454)$ & 0.071 \\
PSMD6 (rs831571) & TT + TC vs. CC & 4.762 & $1.519(1.044,2.212)$ & 0.044 \\
\hline
\end{tabular}

Genetic variables were adjusted for waist circumference, SBP, duration of DM, albuminuria categories, HbA1c, total cholesterol, and triglycerides as covariates. $p$ values were false discovery rate (FDR) corrected. Post-hoc power analysis: FTO (rs8050136), power = 0.657; PSMD6 (rs831571), power = 0.647. S.E.: standard error; OR: odds ratio; CI: confidence interval.

Table 4. Backward stepwise regression analysis on the association between SNPs rs8050136 and rs831571 on diabetic retinopathy risk using the recessive model, adjusted for DM medication usage.

\begin{tabular}{cccc}
\hline Variables & S.E. & OR (95\% CI) & $p$-Value \\
\hline DM medications & & & 0.003 \\
Insulin & 0.262 & $2.609(1.561,4.360)$ & 0.043 \\
GLP-1 receptor agonists & 0.499 & $2.838(1.067,7.553)$ & 0.041 \\
genetics & & & 0.076 \\
FTO (rs8050136) & 4.780 & $5.851(1.201,28.510)$ & \\
PSMD6 (rs831571) & 3.144 & $1.414(0.964,2.074)$ & \\
\hline
\end{tabular}

Genetic variables adjusted for waist circumference, systolic blood pressure (SBP), duration of diabetes mellitus (DM), albuminuria categories, $\mathrm{HbA1c}$, total cholesterol, triglycerides, DPP-4 inhibitors, sulfonylureas, glinides, SGLT-2 inhibitors, $\alpha$-glucosidase inhibitors, biguanides, thiazolidinediones, insulin, GLP-1 receptor agonists. $p$ values were false discovery rate (FDR) corrected. Post-hoc power analysis: FTO $($ rs8050136), power $=0.716 ;$ PSMD6 (rs831571), power = 0.472. S.E.: standard error; OR: odds ratio; CI: confidence interval.

The associations of risk allele frequency with DR severity are shown in Figure 2. Both FTO (rs8050136) and PSMD6 (rs831571) demonstrated a trend of higher DR severity levels in subjects with risk alleles. Figure 3 shows that the prevalence of VTDR was higher in patients carrying more risk alleles of FTO (rs8050136) at 12.6\%, 13.5\%, and 25.0\%, 
respectively $(p=0.448)$. For PSMD6 (rs831571), patients carrying more risk alleles had a higher trend of PDR prevalence (Figure 4a). Carriers of the $C$ allele showed a significant increasing trend of PDR prevalence in patients without using thiazolidinedones $(p=0.015)$ (Figure $4 b$ ).
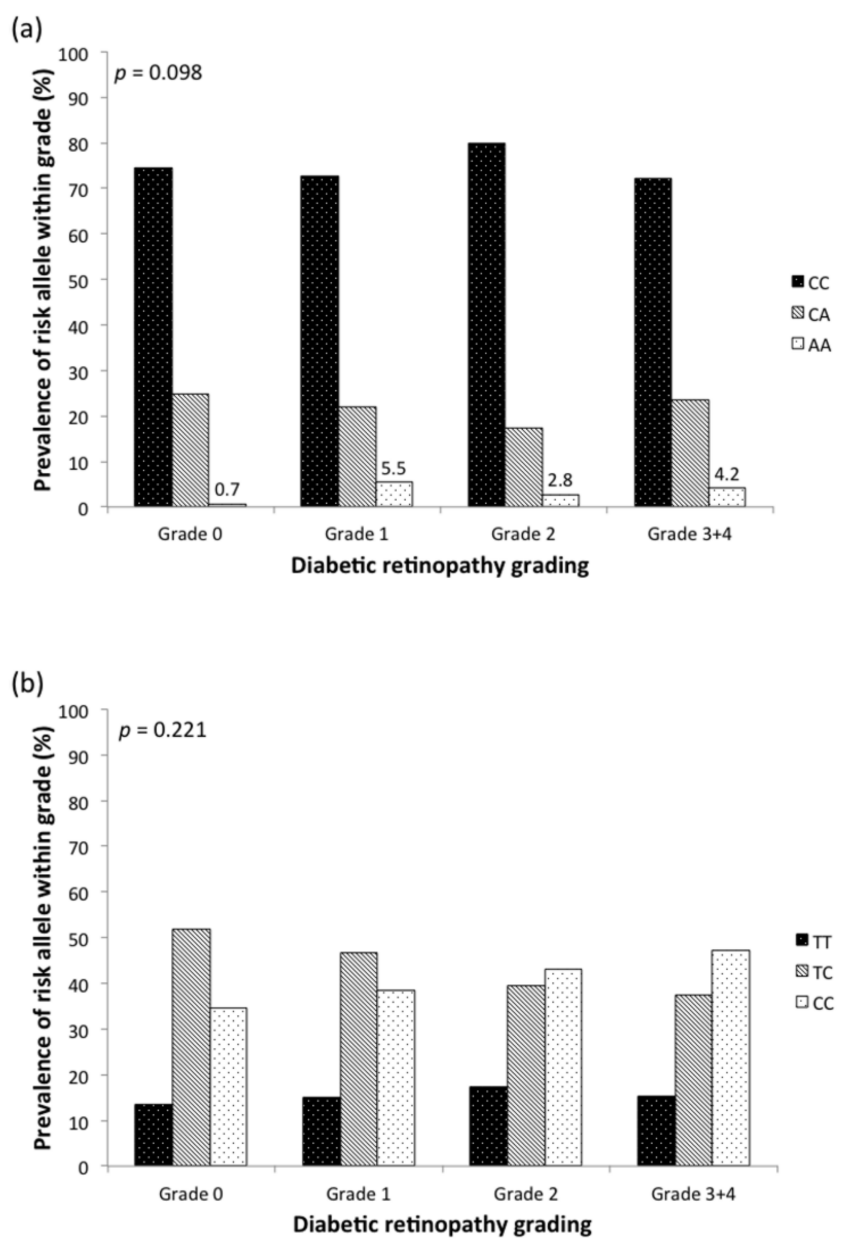

Figure 2. Comparison of the prevalence of risk alleles in different DR stages in T2DM patients (a) FTO rs8050136 (b) PSMD6 rs831571. DR grading as follows: Grade 0, no DR; grade 1, mild NPDR; grade 2, moderate NPDR; grade 3 , severe NPDR; grade 4, PDR. $p$ value from chi-square test.

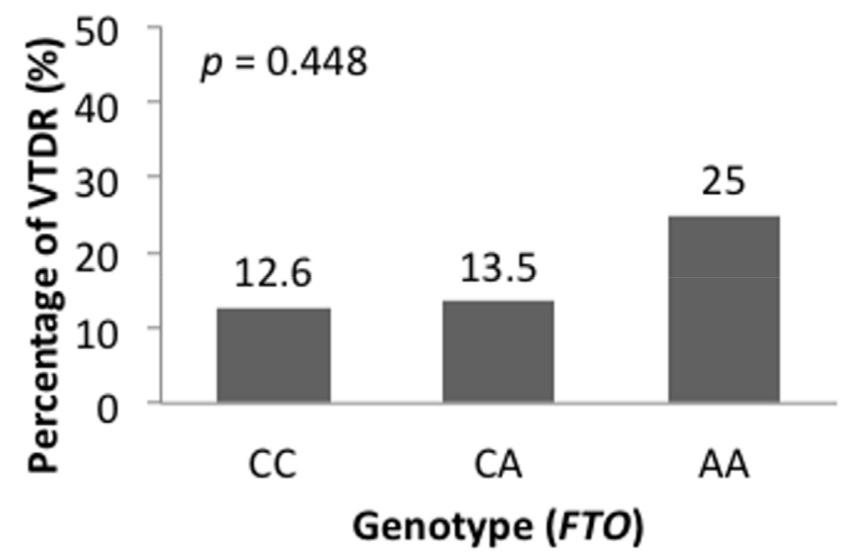

Figure 3. The prevalence of vision-threatening DR (including severe NDPR and PDR) in T2DM patients with genotypic variants (CC, CA, AA) of FTO rs8050136. $p$ value from chi-square test. 
(a)

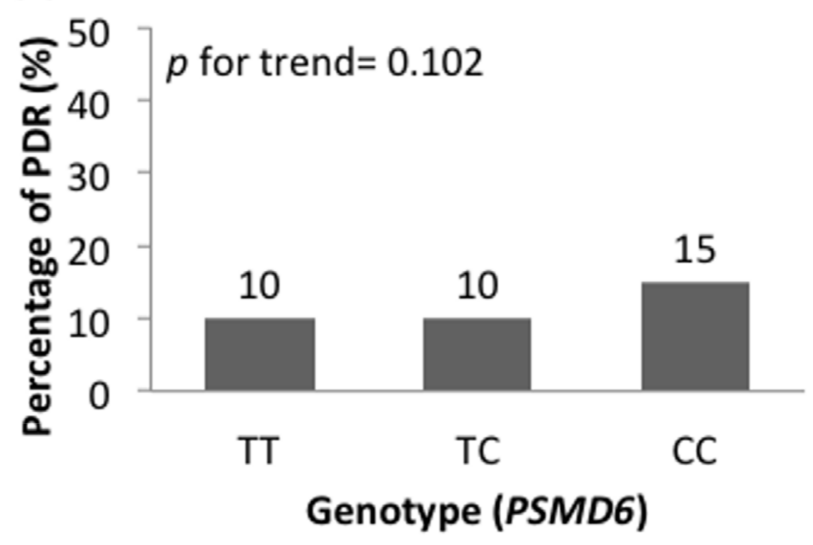

(b)

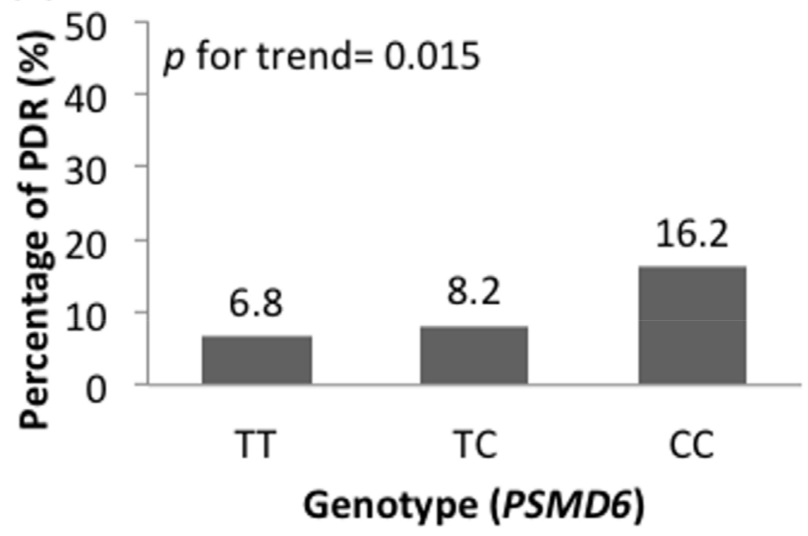

Figure 4. The effect of thiazolidinediones (TZD) usage on the prevalence of proliferative diabetic retinopathy (PDR) in T2DM patients of PSMD6 rs831571 variant. The bar chart indicates the PDR prevalence in patients (a) without using and (b) using TZD according to genotype of PSMD6.

We also investigated the association of $F T O$ (rs8050136) with obesity. Our results indicated a trend $(p=0.087)$ between DR and waist circumference under per-allele comparison of rs8050136, despite being insignificant.

\subsection{Validation of Risk Prediction Model}

To verify the discriminatory accuracy of our genetic risk prediction model with conventional DR risk factors, we evaluated the different AUC estimates in nongenetic, genetic, and combined risk prediction models. The nongenetic risk prediction model was determined from known nongenetic risk factors for DR, including $\mathrm{HbA1c}$, DM duration, waist circumference, SBP, albuminuria categories, dyslipidemia et al. The AUC for HbA1c was 0.631 (95\% CI, 0.584-0.678), which increased to 0.685 (95\% CI, 0.640-0.730) after including DM duration. The AUC for all nongenetic risk factors (waist circumference, SBP, DM duration, $\mathrm{HbA1c}$, albuminuria categories, cholesterol, triglycerides) was 0.704 (95\% CI, 0.661-0.748) (Figure 5). 


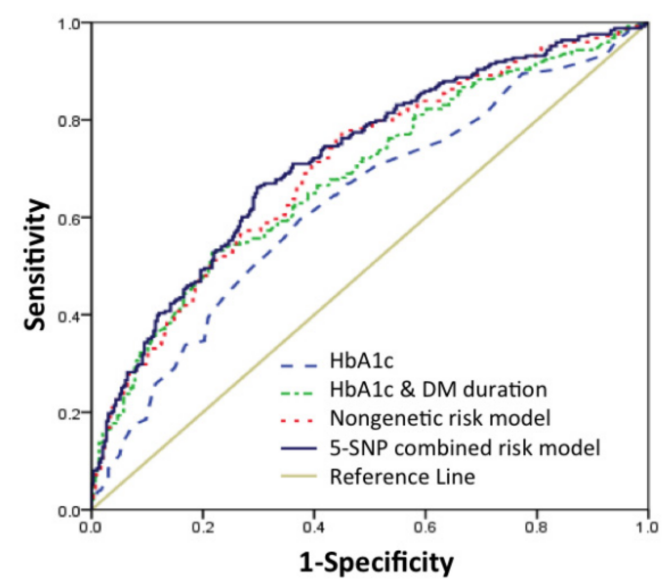

Figure 5. Receiver operating characteristic (ROC) curves and area under the curve (AUC) in diabetic patients. Blue line: $\mathrm{HbA1c}$ risk score $(\mathrm{AUC}=0.631)$; green line: $\mathrm{HbA} 1 \mathrm{c}$ and DM duration risk model $(\mathrm{AUC}=0.685)$; red line: nongenetic risk model (AUC $=0.704)$; navy line: 5-SNP combined risk model $(A U C=0.722)$. The beige line represents the reference line (no discrimination).

We added SNPs to the nongenetic risk prediction model on an individual basis, in order of the SNPs with the greatest OR in logistic regression analysis (Table S1), in order to build a combined risk prediction model using the most practical method. Subsequently, we evaluated the AUC after adding each SNP (Figure 6). The discriminative accuracy of the predictive model went up with inclusion of each SNP, up to a total 5 SNPs. These SNPs were FTO (rs8050136), PSMD6 (rs831571), TCF7L2 (rs7901695), KCNJ11 (rs5219), KCNQ1 (rs2237892) in order of addition. This 5-SNP combined risk prediction model had an AUC of 0.722 (95\% CI, 0.679-0.764) (Figure 5).

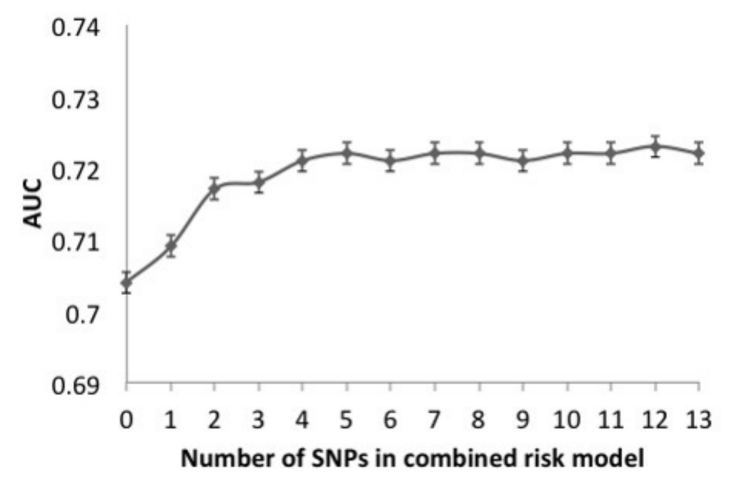

Figure 6. Area under the ROC of nongenetic and genetic combined risk model based on increasing number of SNPs. SNPs were added in order of the odds ratio (see Table 2); starting with FTO based on 1 SNP and ending with SLC22A1 (13 SNPs). Nongenetic risk model (0 SNP) was based on SBP, $\mathrm{DM}$ duration, waist circumference, $\mathrm{HbA1c}$, albuminuria categories, total cholesterol, high-density lipoprotein, low-density lipoprotein, triglycerides. ROC, receiver operating characteristic curve; AUC, area under ROC curve.

In addition, we assessed the discriminatory ability of our model in different subgroups. After classifying the DR subjects into UACR $<30 \mathrm{mg} / \mathrm{g}$ (albuminuria category A1, $n=295$ ) and UACR $\geq 30 \mathrm{mg} / \mathrm{g}$ (albuminuria categories A2 and A3, $n=256$ ) (Figure 7a,b), the combined nongenetic and genetic risk prediction model (AUC $=0.731 ; 95 \%$ CI, $0.669-0.793$ ) showed a larger increase in discrimination ability than the conventional nongenetic risk prediction model (AUC $=0.693 ; 95 \%$ CI, $0.628-0.757$ ) in the group with albuminuria categories A2 and A3 than that in A1 group. Moreover, in diabetic patients with well glycemic control (HbA1c $<8 \%, n=402)$ and poor glycemic control $(\mathrm{HbA} 1 \mathrm{c} \geq 8 \%, n=149)$ (Figure 7c,d) [31], we found that the discrimination ability increased to a greater extent in 
subjects with poor glycemic control when comparing between the 5-SNP combined risk prediction model (AUC $=0.746 ; 95 \% \mathrm{CI}, 0.666-0.825$ ) and nongenetic risk prediction model (AUC $=0.659 ; 95 \%$ CI, $0.570-0.748)$ alone.

(a)

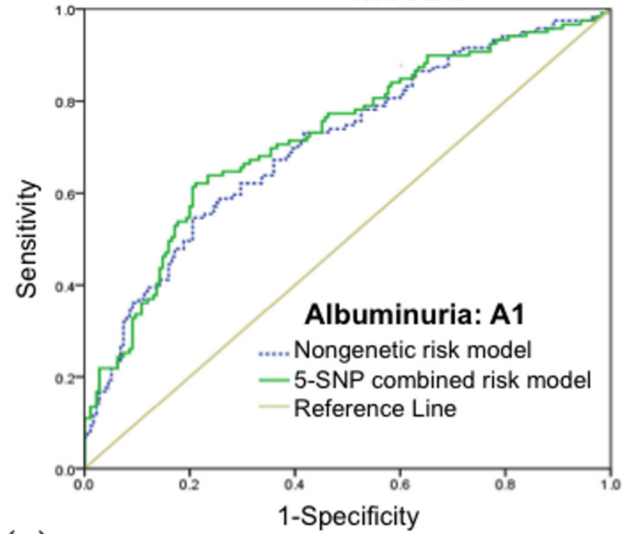

(c)

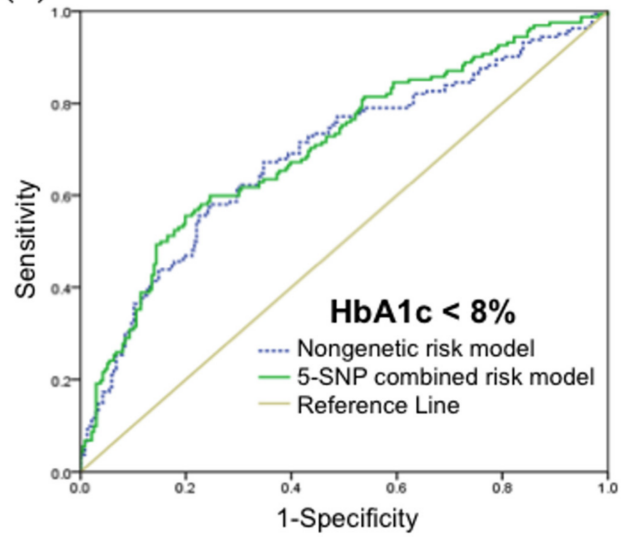

(b)

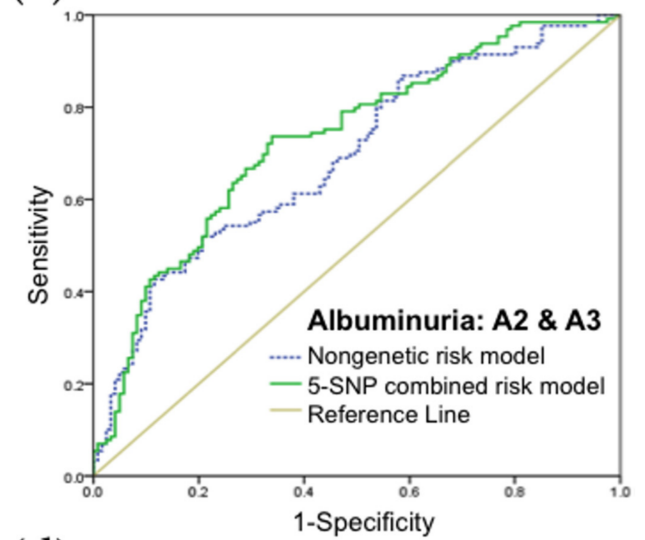

(d)

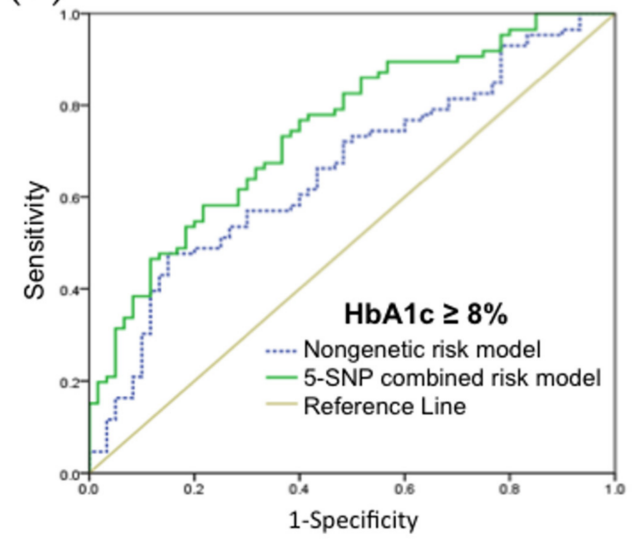

Figure 7. Receiver operating characteristic (ROC) curves and area under the curve (AUC) in different diabetic subgroups; (a) diabetic patients in albuminuria category A1. Blue line: nongenetic risk model (AUC $=0.708)$; green line: 5-SNP combined risk model (AUC $=0.724)$; $(\mathbf{b})$ diabetic patients in albuminuria category A2 and A3. Blue line: nongenetic risk model (AUC $=0.693$ ); green line: 5-SNP combined risk model (AUC $=0.731$ ); (c) diabetic patients with $\mathrm{HbA1c}$ levels $<8 \%$. Blue line: nongenetic risk model (AUC $=0.690$ ); green line: 5-SNP combined risk model (AUC $=0.707)$; $(\mathbf{d})$ diabetic patients with $\mathrm{HbA1c}$ levels $\geq 8 \%$. Blue line: nongenetic risk model (AUC $=0.659$ ); green line: 5 -SNP combined risk model (AUC $=0.746)$. The beige line represents the reference line (no discrimination).

\section{Discussion}

In this study, we presented novel genetic T2DM related genetic variants associated with DR risk and progression and established a comprehensive multifactorial prediction model. Of the 13 T2DM genetic loci, we found FTO rs8050136 and PSMD6 rs831571 as genetic markers of DR development, independent of traditional risk factors. In our analysis, a risk prediction model based on the 5 SNPs with the greatest OR appeared to distinguish patients with and without DR similarly, as well as the initial genetic risk model based on 13 SNPs. Our findings may provide a practical method for screening T2DM patients who are susceptible of DR, and a promising contribution to the understanding of the mechanisms underlying DR progression.

Diabetes is known for its microvascular and macrovascular complications that contribute to long-term damage and failure of organ systems. Microangiopathy is associated with increased damage in different organs, thus promoting retinopathy, nephropathy, and 
neuropathy. Oxidative stress and inflammation on vascular alterations were found to affect DR development $[32,33]$. Obesity plays an important role in microvascular dysfunction as well [34]. Previous studies have indicated the importance of obesity-related factors and oxidative stress in DR risk. Taken together, these theories imply that microvascular damage and metabolic physiology in hyperglycemia may form a vicious cycle with multiorgan consequences. Given the high morbidity and mortality associated with T2D complications, identifying genetic risk factors for T2D complications is critical.

We found the DR risk increased significantly in patients with the FTO (rs8050136) genetic polymorphism, even after adjustment for traditional risk factors and different DM medications use. Subjects with risk allele (A) of this variant showed a trend for DR progression. In both Western and Chinese populations, the FTO polymorphism rs8050136 has been linked to obesity markers (BMI, waist, and hip circumference) and glucose homeostasis [35-37]. Bravard et al. demonstrated that FTO mRNA and protein levels in skeletal muscle from T2DM patients increased, implying that FTO may have a role in oxidative metabolism [38]. Interestingly, variants in the FTO gene correlated to a decrease in the presence of obesity [39], which is a risk factor for retinopathy [40]. Our subgroup analysis implied that rs8050136 risk allele (A) and obesity may be correlated. Although the number of risk alleles in rs8050136 was related to increased waist circumference, the results were insignificant possibly due to the limited genotype population. Therefore, the exact mechanism underlying the FTO function and DR is still illusive and demands further research.

The encoded protein of the PSMD6 gene is a member of the ubiquitin-proteasome system and a subunit of the 26S proteasome (UPS) [41]. The UPS is the foundation of regulating cell cycle, protein quality control, and differentiation and numerous signal transduction pathways. In previous GWAS studies, the PSMD6 rs831571 variation was discovered to be a T2DM susceptibility locus [42,43]. In the retina and kidney, Aghdam and Sheibaani have expressed that hyperglycemia and oxidative stress modulate UPS activity and altered UPS activity in diabetes may give rise to the formation of retinal microvascular complications [44]. The PSMD6 variation rs831571 was shown to be strongly linked to the development of DR in our study, but became insignificant after adjustment for DM medication use. Previously, in a Chinese cohort, the PSMD6 variant rs831571 was observed to be closely related to the therapeutic properties of rosiglitazone. It was inferred that as the 265 proteasome is a subunit of UPS, SNPs in the PSMD6 gene may alter the oral antidiabetic drug effects due to its impact on insulin secretion and sensitivity [45]. Nevertheless, the association between PSMD6 and rosiglitazone use cannot explain the effect of different genotype of PSMD6 on DR in our cohort. After excluding thiazolidinedione users, we discovered the risk allele (C) of PSMD6 (rs831571) to be correlated with DR severity progression, more specifically, the advancement into PDR.

The development of DR may be related to high levels of inflammation and insulin resistance in diabetic individuals with abdominal obesity [46-48]. Besides FTO polymorphisms, KCNJ11 rs5219, KCNQ1 rs2237892, and TCF7L2 rs7901695 variations have previously been linked to obesity in patients with T2DM. In Caucasians and East Asian groups, the KCNJ11 rs5219 polymorphism has been identified as a risk factor for developing T2DM. It can impact the insulin secretion pathway by decreasing ATP sensitivity of the KATP channel, resulting in suppression of insulin secretion. This variant also plays a major role in blood pressure and HbA1c levels in diabetes [49]. By impairing $\beta$-cell function, KCNQ1 confers a risk for T2DM, and was realized to be strongly linked to a greater likelihood of T2DM in European and East Asian populations [50,51]. In terms of diabetes complications, it was suggested that the KCNQ1 rs2237892 variant may contribute to susceptibility in macrovascular disease, diabetic nephropathy, and diabetic retinopathy [52,53]. In previous studies, there was a significant association of KCNQ1 rs2237892 and TCF7L2 rs7901695 variants with impaired lipid parameters [54,55]. Although hyperglycemia is the main initiator in DR, dyslipidemia is also associated with the development of this disease [56]. In an animal experimental model, obesity was found to alter hyperglycemia-induced epigenetic 
modifications, accelerating mitochondrial damage and DR [57]. Despite the fact that the associations of KCNJ11 rs5219, KCNQ1 rs2237892, and TCF7L2 rs7901695 with DR are not significant in our data, we believe in a more long-term longitudinal study they may still influence the development and progression of DR according to our predictive model.

We established a risk prediction model based on the SNPs that were most consistently linked to T2DM and discovered a "dose-response" relationship between the risk prediction model and DR - to an extent, the more SNPs involved, the greater the risk of DR. The risk prediction model based on the five SNPs with the highest ORs in our data appeared to discriminate between patients with and without DR equally effectively as the initial model constructed on the selected 13 SNPs. The AUC increased to 0.722 by combining the 5-SNP genetic risk prediction model with the nongenetic risk prediction model, demonstrating good diagnostic accuracy. As the capacity of outpatient clinic follow-up may be inadequate, precise discrimination may help to establish a personalized schedule for fundus follow-up to fulfill the difference between those at greater and lower risk of developing diabetic retinopathy. We investigated in-depth the extent to which risk prediction model can enhance the accuracy of ROC curves for retinopathy risk assessment. Regarding diabetic patients with albuminuria, the 5-SNP genetic risk prediction model (AUC $=0.731$ ) performed better than conventional prediction model $(A U C=0.693)$ alone. In subgroup analysis on the degree of glycemic control, the proportion of discriminative accuracy increased with a larger amount in persons with poor glycemic control. In brief, our 5-SNP prediction model may have greater potential clinical value in certain diabetic populations. In patients with diabetic nephropathy (UACR $\geq 30 \mathrm{mg} / \mathrm{g}$ ) or insufficient glycemic control ( $\mathrm{HbA} 1 \mathrm{c}$ levels $\geq 8 \%$ ), shortened intervals between fundus examinations and intensive diabetes self-management education should be arranged.

The strengths of this study included a comprehensive systemic and medication history assessment, with standardized assessment of DR based on retinal photographs. We also recognize some limitations in our study. Our study population only consisted of Asian ethnicities and the patient number was relatively limited, thus further validation of the risk prediction model in other populations of different ethnicities may be needed. Although the excluded population revealed significance in wider waist circumference and were older when compared to our included subject population, we believe the features may be associated. Elderly diabetic patients are more likely to have a longer duration of DM and wider waist circumferences and reduced ability in completing all aspects required in the study. Lastly, due to this being a cross-sectional study, a long-term longitudinal study may be necessary on further identification of SNP genotypes in terms of DR progression.

\section{Conclusions}

Identification of persons at risk of DR development and progression is of paramount importance in diabetic populations. Our study provides evidence of a novel association between FTO rs8050136 and PSMD6 rs831571 polymorphisms with DR development. The predictive value of our combined nongenetic and genetic risk prediction model may aid in better identifying a subset of patients at greater risk of DR in clinical practice.

Supplementary Materials: The following are available online at https://www.mdpi.com/article/10 .3390/jpm11080689/s1, Table S1: Multiple analysis of SNPs on DR risk with logistic regression using enter method.

Author Contributions: Conceptualization, J.J.L., P.-W.W.; data curation, Y.-T.H., J.-J.L., F.-C.S., S.W.W., P.-W.W., Y.-J.C.; formal analysis, Y.-T.H., J.-J.L.; supervision, F.-C.S., S.-W.W., P.-W.W.; writingoriginal draft, Y.-T.H.; writing — review and editing F.-C.S., S.-W.W., P.-W.W., Y.-J.C., J.-J.L. All authors have read and agreed to the published version of the manuscript.

Funding: This work was supported by the Chang Gung Memorial Hospital under grant [CORPG8F1001, CORPG8F1002 and CORPG8F1003]. 
Institutional Review Board Statement: The study was conducted according to the guidelines of the Declaration of Helsinki, and approved by the Institutional Review Board/Ethics Committee from the Committee of Medical Ethics and Human Experiments of CGMH, Taiwan (reference No. 201601206B0C501).

Informed Consent Statement: Informed consent was obtained from all subjects involved in the study.

Data Availability Statement: Data is fully available upon reasonable request to corresponding author.

Acknowledgments: The authors thank the Biostatistics Center, Kaohsiung Chang Gung Memorial Hospital.

Conflicts of Interest: The authors declare no conflict of interest.

\section{References}

1. Ruta, L.M.; Magliano, D.J.; LeMesurier, R.; Taylor, H.R.; Zimmet, P.Z.; Shaw, J.E. Prevalence of diabetic retinopathy in Type 2 diabetes in developing and developed countries. Diabet. Med. 2013, 30, 387-398. [CrossRef]

2. Flaxman, S.R.; Bourne, R.R.A.; Resnikoff, S.; Ackland, P.; Braithwaite, T.; Cicinelli, M.V.; Das, A.; Jonas, J.B.; Keeffe, J.; Kempen, J.H.; et al. Global causes of blindness and distance vision impairment 1990-2020: A systematic review and meta-analysis. Lancet Glob. Health 2017, 5, e1221-e1234. [CrossRef]

3. Ting, D.S.; Cheung, G.C.; Wong, T.Y. Diabetic retinopathy: Global prevalence, major risk factors, screening practices and public health challenges: A review. Clin. Exp. Ophthalmol. 2016, 44, 260-277. [CrossRef]

4. Cheung, N.; Mitchell, P.; Wong, T.Y. Diabetic retinopathy. Lancet 2010, 376, 124-136. [CrossRef]

5. Federation, I.D. IDF Diabetes Atlas, 9th ed.; International Diabetes Federation: Brussels, Belgium, 2019.

6. Liu, W.J.; Lee, L.T.; Yen, M.F.; Tung, T.H.; Williams, R.; Duffy, S.W.; Chen, T.H. Assessing progression and efficacy of treatment for diabetic retinopathy following the proliferative pathway to blindness: Implication for diabetic retinopathy screening in Taiwan. Diabet Med. 2003, 20, 727-733. [CrossRef]

7. World Health Organization. Strengthening Diagnosis and Treatment of Diabetic Retinopathy in the South-East Asia Region; World Health Organization, Regional Office for South-East Asia: New Delhi, India, 2020. Available online: https:/ /apps.who.int/iris/ bitstream/handle/10665/334224/9789290227946-eng.pdf (accessed on 18 June 2021).

8. Das, T.; Takkar, B.; Sivaprasad, S.; Thanksphon, T.; Taylor, H.; Wiedemann, P.; Nemeth, J.; Nayar, P.D.; Rani, P.K.; Khandekar, R. Recently updated global diabetic retinopathy screening guidelines: Commonalities, differences, and future possibilities. Eye 2021, 1-14. [CrossRef]

9. Storey, P.P.; Murchison, A.P.; Pizzi, L.T.; Hark, L.A.; Dai, Y.; Leiby, B.E.; Haller, J.A. Impact of physician communication on diabetic eye examination adherence: Results From a Retrospective Cohort Analysis. Retina 2016, 36, 20-27. [CrossRef] [PubMed]

10. Ogunyemi, O.; Kermah, D. Machine Learning Approaches for Detecting Diabetic Retinopathy from Clinical and Public Health Records. AMIA Annu. Symp. Proc. 2015, 2015, 983-990. [PubMed]

11. Ogunyemi, O.I.; Gandhi, M.; Tayek, C. Predictive Models for Diabetic Retinopathy from Non-Image Teleretinal Screening Data. AMIA Summits Transl. Sci. Proc. 2019, 2019, 472-477. [PubMed]

12. Yau, J.W.; Rogers, S.L.; Kawasaki, R.; Lamoureux, E.L.; Kowalski, J.W.; Bek, T.; Chen, S.J.; Dekker, J.M.; Fletcher, A.; Grauslund, J.; et al. Global prevalence and major risk factors of diabetic retinopathy. Diabetes Care 2012, 35, 556-564. [CrossRef]

13. Vujosevic, S.; Aldington, S.J.; Silva, P.; Hernández, C.; Scanlon, P.; Peto, T.; Simó, R. Screening for diabetic retinopathy: New perspectives and challenges. Lancet Diabetes Endocrinol. 2020, 8, 337-347. [CrossRef]

14. Lachin, J.M.; Genuth, S.; Nathan, D.M.; Zinman, B.; Rutledge, B.N. Effect of glycemic exposure on the risk of microvascular complications in the diabetes control and complications trial-Revisited. Diabetes 2008, 57, 995-1001. [CrossRef]

15. Han, J.; Lando, L.; Skowronska-Krawczyk, D.; Chao, D.L. Genetics of Diabetic Retinopathy. Curr. Diabetes Rep. 2019, 19, 67. [CrossRef] [PubMed]

16. Sharma, A.; Valle, M.L.; Beveridge, C.; Liu, Y.; Sharma, S. Unraveling the role of genetics in the pathogenesis of diabetic retinopathy. Eye 2019, 33, 534-541. [CrossRef] [PubMed]

17. Fan, W.Y.; Gu, H.; Yang, X.F.; She, C.Y.; Liu, X.P.; Liu, N.P. Association of candidate gene polymorphisms with diabetic retinopathy in Chinese patients with type 2 diabetes. Int. J. Ophthalmol. 2020, 13, 301-308. [CrossRef]

18. Irace, C.; Scarinci, F.; Scorcia, V.; Bruzzichessi, D.; Fiorentino, R.; Randazzo, G.; Scorcia, G.; Gnasso, A. Association among low whole blood viscosity, haematocrit, haemoglobin and diabetic retinopathy in subjects with type 2 diabetes. Br. J. Ophthalmol. 2011, 95, 94-98. [CrossRef] [PubMed]

19. Wang, R.; Miao, Z.; Liu, T.; Liu, M.; Grdinovac, K.; Song, X.; Liang, Y.; Delen, D.; Paiva, W. Derivation and Validation of Essential Predictors and Risk Index for Early Detection of Diabetic Retinopathy Using Electronic Health Records. J. Clin. Med. 2021, 10, 1473. [CrossRef] [PubMed]

20. Chong, Y.H.; Fan, Q.; Tham, Y.C.; Gan, A.; Tan, S.P.; Tan, G.; Wang, J.J.; Mitchell, P.; Wong, T.Y.; Cheng, C.Y. Type 2 Diabetes Genetic Variants and Risk of Diabetic Retinopathy. Ophthalmology 2017, 124, 336-342. [CrossRef] [PubMed]

21. Liao, W.L.; Lin, J.M.; Chen, W.L.; Hsieh, M.C.; Wu, C.M.; Chang, Y.W.; Huang, Y.C.; Tsai, F.J. Multilocus genetic risk score for diabetic retinopathy in the Han Chinese population of Taiwan. Sci. Rep. 2018, 8, 1-9. [CrossRef]

22. Marathe, P.H.; Gao, H.X.; Close, K.L. American Diabetes Association Standards of Medical Care in Diabetes 2017. J. Diabetes 2017, 9, 320-324. [CrossRef] [PubMed] 
23. Whelton, P.K.; Carey, R.M.; Aronow, W.S.; Casey, D.E., Jr.; Collins, K.J.; Dennison Himmelfarb, C.; DePalma, S.M.; Gidding, S.; Jamerson, K.A.; Jones, D.W.; et al. 2017 ACC/AHA/AAPA/ABC/ACPM/AGS/APhA/ASH/ASPC/NMA/PCNA Guideline for the Prevention, Detection, Evaluation, and Management of High Blood Pressure in Adults: A Report of the American College of Cardiology/American Heart Association Task Force on Clinical Practice Guidelines. J. Am. Coll. Cardiol. 2018, 71, e127-e248. [CrossRef] [PubMed]

24. Pettitt, D.J.; Talton, J.W.; Liese, A.D.; Liu, L.L.; Crimmins, N.; West, N.A.; D'Agostino, R.B., Jr.; Kahn, H.S.; SEARCH for Diabetes in Youth Study Group. Comparison of two waist circumference measurement protocols: The SEARCH for diabetes in youth study. Pediatric Obes. 2012, 7, e81-e85. [CrossRef] [PubMed]

25. Stevens, P.E.; Levin, A. Evaluation and Management of Chronic Kidney Disease: Synopsis of the Kidney Disease: Improving Global Outcomes 2012 Clinical Practice Guideline. Ann. Intern. Med. 2013, 158, 825-830. [CrossRef] [PubMed]

26. Wilkinson, C.P.; Ferris, F.L., 3rd; Klein, R.E.; Lee, P.P.; Agardh, C.D.; Davis, M.; Dills, D.; Kampik, A.; Pararajasegaram, R.; Verdaguer, J.T. Proposed international clinical diabetic retinopathy and diabetic macular edema disease severity scales. Ophthalmology 2003, 110, 1677-1682. [CrossRef]

27. Wong, T.Y.; Cheung, N.; Tay, W.T.; Wang, J.J.; Aung, T.; Saw, S.M.; Lim, S.C.; Tai, E.S.; Mitchell, P. Prevalence and risk factors for diabetic retinopathy: The Singapore Malay Eye Study. Ophthalmology 2008, 115, 1869-1875. [CrossRef]

28. Ahmad, A.; Sundquist, K.; Palmér, K.; Svensson, P.J.; Sundquist, J.; Memon, A.A. Risk prediction of recurrent venous thromboembolism: A multiple genetic risk model. J. Thromb. Thrombolysis 2019, 47, 216-226. [CrossRef]

29. de Haan, H.G.; Bezemer, I.D.; Doggen, C.J.; Le Cessie, S.; Reitsma, P.H.; Arellano, A.R.; Tong, C.H.; Devlin, J.J.; Bare, L.A.; Rosendaal, F.R.; et al. Multiple SNP testing improves risk prediction of first venous thrombosis. Blood 2012, 120, 656-663. [CrossRef]

30. Hanley, J.A.; McNeil, B.J. The meaning and use of the area under a receiver operating characteristic (ROC) curve. Radiology 1982, 143, 29-36. [CrossRef] [PubMed]

31. Ferris, F.L., III; Nathan, D.M. Preventing Diabetic Retinopathy Progression. Ophthalmology 2016, 123, 1840-1842. [CrossRef] [PubMed]

32. Rodríguez, M.L.; Pérez, S.; Mena-Mollá, S.; Desco, M.C.; Ortega, Á.L. Oxidative Stress and Microvascular Alterations in Diabetic Retinopathy: Future Therapies. Oxid. Med. Cell. Longev. 2019, 2019, 4940825. [CrossRef]

33. Li, H.; Yu, X.; Zheng, B.; Ding, S.; Mu, Z.; Guo, L. Early neurovascular changes in the retina in preclinical diabetic retinopathy and its relation with blood glucose. BMC Ophthalmol. 2021, 21, 1-9. [CrossRef]

34. Stehouwer, C.D.A. Microvascular Dysfunction and Hyperglycemia: A Vicious Cycle with Widespread Consequences. Diabetes 2018, 67, 1729-1741. [CrossRef]

35. Zhao, N.N.; Dong, G.P.; Wu, W.; Wang, J.L.; Ullah, R.; Fu, J.F. FTO gene polymorphisms and obesity risk in Chinese population: A meta-analysis. World J. Pediatr. 2019, 15, 382-389. [CrossRef]

36. Wu, J.; Xu, J.; Zhang, Z.; Ren, J.; Li, Y.; Wang, J.; Cao, Y.; Rong, F.; Zhao, R.; Huang, X.; et al. Association of FTO polymorphisms with obesity and metabolic parameters in Han Chinese adolescents. PLoS ONE 2014, 9, e98984. [CrossRef]

37. Xiao, S.; Zeng, X.; Quan, L.; Zhu, J. Correlation between polymorphism of FTO gene and type 2 diabetes mellitus in Uygur people from northwest China. Int. J. Clin. Exp. Med. 2015, 8, 9744-9750. [PubMed]

38. Bravard, A.; Lefai, E.; Meugnier, E.; Pesenti, S.; Disse, E.; Vouillarmet, J.; Peretti, N.; Rabasa-Lhoret, R.; Laville, M.; Vidal, H.; et al. FTO is increased in muscle during type 2 diabetes, and its overexpression in myotubes alters insulin signaling, enhances lipogenesis and ROS production, and induces mitochondrial dysfunction. Diabetes 2011, 60, 258-268. [CrossRef]

39. Franceschini, N.; Shara, N.M.; Wang, H.; Voruganti, V.S.; Laston, S.; Haack, K.; Lee, E.T.; Best, L.G.; MacCluer, J.W.; Cochran, B.J.; et al. The association of genetic variants of type 2 diabetes with kidney function. Kidney Int. 2012, 82, 220-225. [CrossRef]

40. Mottl, A.K.; Kwon, K.S.; Garg, S.; Mayer-Davis, E.J.; Klein, R.; Kshirsagar, A.V. The association of retinopathy and low GFR in type 2 diabetes. Diabetes Res. Clin. Pract. 2012, 98, 487-493. [CrossRef]

41. Wang, X.; Chen, C.-F.; Baker, P.R.; Chen, P.-1.; Kaiser, P.; Huang, L. Mass Spectrometric Characterization of the Affinity-Purified Human 26S Proteasome Complex. Biochemistry 2007, 46, 3553-3565. [CrossRef]

42. Zhang, S.; Jamaspishvili, E.; Tong, H.; Chen, Y.; Zhou, Z.; Sun, L.; Kazakova, E.; Hong, Q. East Asian Genome-wide association study derived loci in relation to type 2 diabetes in the Han Chinese population. Acta Biochim. Pol. 2019, 66, 159-165. [CrossRef] [PubMed]

43. Cho, Y.S.; Chen, C.H.; Hu, C.; Long, J.; Ong, R.T.; Sim, X.; Takeuchi, F.; Wu, Y.; Go, M.J.; Yamauchi, T.; et al. Meta-analysis of genome-wide association studies identifies eight new loci for type 2 diabetes in east Asians. Nat. Genet. 2011, 44, 67-72. [CrossRef]

44. Aghdam, S.Y.; Sheibani, N. The ubiquitin-proteasome system and microvascular complications of diabetes. J. Ophthalmic Vis. Res. 2013, 8, 244-256.

45. Chen, M.; Hu, C.; Zhang, R.; Jiang, F.; Wang, J.; Peng, D.; Tang, S.; Sun, X.; Yan, J.; Wang, S.; et al. A variant of PSMD6 is associated with the therapeutic efficacy of oral antidiabetic drugs in Chinese type 2 diabetes patients. Sci. Rep. 2015, 5, 1-7. [CrossRef]

46. Zhou, Y.; Zhang, Y.; Shi, K.; Wang, C. Body mass index and risk of diabetic retinopathy: A meta-analysis and systematic review. Medicine 2017, 96, e6754. [CrossRef]

47. Panagiotakos, D.B.; Pitsavos, C.; Yannakoulia, M.; Chrysohoou, C.; Stefanadis, C. The implication of obesity and central fat on markers of chronic inflammation: The ATTICA study. Atherosclerosis 2005, 183, 308-315. [CrossRef] 
48. Fujimoto, W.Y.; Abbate, S.L.; Kahn, S.E.; Hokanson, J.E.; Brunzell, J.D. The visceral adiposity syndrome in Japanese-American men. Obes. Res. 1994, 2, 364-371. [CrossRef] [PubMed]

49. Haghvirdizadeh, P.; Mohamed, Z.; Abdullah, N.A.; Haghvirdizadeh, P.; Haerian, M.S.; Haerian, B.S. KCNJ11: Genetic Polymorphisms and Risk of Diabetes Mellitus. J. Diabetes Res. 2015, 2015, 908152. [CrossRef] [PubMed]

50. Rosengren, A.H.; Braun, M.; Mahdi, T.; Andersson, S.A.; Travers, M.E.; Shigeto, M.; Zhang, E.; Almgren, P.; Ladenvall, C.; Axelsson, A.S.; et al. Reduced Insulin Exocytosis in Human Pancreatic $\beta$-Cells With Gene Variants Linked to Type 2 Diabetes. Diabetes 2012, 61, 1726-1733. [CrossRef]

51. Unoki, H.; Takahashi, A.; Kawaguchi, T.; Hara, K.; Horikoshi, M.; Andersen, G.; Ng, D.P.K.; Holmkvist, J.; Borch-Johnsen, K.; Jørgensen, T.; et al. SNPs in KCNQ1 are associated with susceptibility to type 2 diabetes in East Asian and European populations. Nat. Genet. 2008, 40, 1098-1102. [CrossRef] [PubMed]

52. Zhang, W.; Wang, H.; Guan, X.; Niu, Q.; Li, W. Variant rs2237892 of KCNQ1 Is Potentially Associated with Hypertension and Macrovascular Complications in Type 2 Diabetes Mellitus in A Chinese Han Population. Genom. Proteom. Bioinform. 2015, 13, 364-370. [CrossRef]

53. Rattanatham, R.; Settasatian, N.; Komanasin, N.; Kukongviriyapan, U.; Sawanyawisuth, K.; Intharaphet, P.; Senthong, V.; Settasatian, C. Association of Combined TCF7L2 and KCNQ1 Gene Polymorphisms with Diabetic Micro- and Macrovascular Complications in Type 2 Diabetes Mellitus. Diabetes Metab. J. 2021. [CrossRef]

54. van Vliet-Ostaptchouk, J.V.; van Haeften, T.W.; Landman, G.W.; Reiling, E.; Kleefstra, N.; Bilo, H.J.; Klungel, O.H.; de Boer, A.; van Diemen, C.C.; Wijmenga, C.; et al. Common variants in the type 2 diabetes KCNQ1 gene are associated with impairments in insulin secretion during hyperglycaemic glucose clamp. PLoS ONE 2012, 7, e32148. [CrossRef]

55. Včelák, J.; Vejražková, D.; Vaňková, M.; Lukášová, P.; Bradnová, O.; Hálková, T.; Bešt ák, J.; Andělová, K.; Kvasničková, H.; Hoskovcová, P.; et al. T2D risk haplotypes of the TCF7L2 gene in the Czech population sample: The association with free fatty acids composition. Physiol. Res. 2012, 61, 229-240. [CrossRef] [PubMed]

56. Chew, E.Y.; Klein, M.L.; Ferris, F.L., 3rd; Remaley, N.A.; Murphy, R.P.; Chantry, K.; Hoogwerf, B.J.; Miller, D. Association of elevated serum lipid levels with retinal hard exudate in diabetic retinopathy. Early Treatment Diabetic Retinopathy Study (ETDRS) Report 22. Arch. Ophthalmol. 1996, 114, 1079-1084. [CrossRef]

57. Kowluru, R.A. Retinopathy in a Diet-Induced Type 2 Diabetic Rat Model and Role of Epigenetic Modifications. Diabetes 2020, 69, 689-698. [CrossRef] [PubMed] 